\title{
GPS Modeling for Designing Aerospace Vehicle Navigation
} Systems

John J. Dougherty

TRW System Integration Group

Hossny El-Sherief

TRW System Integration Group

Paniel this Simpn andditional works at: https://engagedscholarship.csuohio.edu/enece_facpub Cleveland State University, d.j.simon@csuohio.eđu

Part of the Navigation, Guidance, Control and Dynamics Commons

Gary Aowhitmer so to this work benefit you? Let us know!

TRW System lntegration Group

Publisher's Statement

(C) 1995 IEEE. Personal use of this material is permitted. Permission from IEEE must be obtained for all other users, including reprinting/ republishing this material for advertising or promotional purposes, creating new collective works for resale or redistribution to servers or lists, or reuse of any copyrighted components of this work in other works.

\section{Original Citation}

Dougherty, J.J.; El-Sherief, H.; Simon, D.J.; Whitmer, G.A. (1995). GPS modeling for designing aerospace vehicle navigation systems. Aerospace and Electronic Systems, IEEE Transactions on, 31(2), 695-705, doi: 10.1109/7.381917.

\section{Repository Citation}

Dougherty, John J.; El-Sherief, Hossny; Simon, Daniel J.; and Whitmer, Gary A., "GPS Modeling for Designing Aerospace Vehicle Navigation Systems" (1995). Electrical Engineering \& Computer Science Faculty Publications. 132.

https://engagedscholarship.csuohio.edu/enece_facpub/132

This Article is brought to you for free and open access by the Electrical Engineering \& Computer Science Department at EngagedScholarship@CSU. It has been accepted for inclusion in Electrical Engineering \& Computer Science Faculty Publications by an authorized administrator of EngagedScholarship@CSU. For more information, please contact library.es@csuohio.edu. 


\section{GPS Modeling for Designing Aerospace Vehicle Navigation} Systems

\author{
JOHN J, DOUGHERTY \\ HOSSNY EL-SHERIEF \\ DANIEL I. SIMON \\ GARY A. WHITMER \\ TRW Systems Integration Group
}

The complexity of the design of a Global Positioning System (GPS) user segment, as well as the performance demanded of the componenis, depends on user requirements such as total navigation accuracy, Other factors, for instance the expected satellite/vehicle geometry or the accuracy of an accompanying inertial navigation system, can also affect the user segment design. Models of GPS measurements are used to predict user segment performance at various levels. Design curves are developed which illustrate the relationship between user requirements, the user segment design, and component performance.

\section{INTRODUCTION}

Because of the versatility provided by its global availability and the passive nature of the user segment, the Global Positioning System (GPS) is being used in a wide range of aerospace applications. Among these are on-board navigators and trajectory references for range safety and for testing inertial navigation systems. GPS is a satellite navigation system developed and maintained by the United States Department of Defense. It includes 24 satellites in semigeosynchronous orbit providing continuous global coverage and excellent navigation accuracy [1].

A GPS user segment comprises the hardware and software employed by the user to obtain navigation information from GPS. The user segment must be designed so that some user performance requirement is met. For a GPS user segment employed as a component of an on-board navigation system, the requirement is usually on the total navigation accuracy. For a GPS user segment as an autonomous navigator, the requirement is usually on the GPS navigation accuracy itself. For a GPS user segment as a trajectory reference for testing inertial navigation systems, the requirement is usually on the ability to achieve test objectives such as estimating the inertial navigation system accuracy $[2,3]$.

GPS user segment designs can be broadly classified into two categories: receiver- and translator-based designs. A GPS receiver processes GPS signals to estimate its own position and velocity. This information can be used directly, or can be combined with other navigation estimates (from an inertial navigation system, for instance) to get a best-estimate of the vehicle position and velocity [4-6]. A GPS receiver must compensate for known measurement errors in real-time [7]. A GPS translator, on the other hand, is a relatively simple device whose function is to frequency shift ("translate") the GPS signals from one frequency band to another, such as a telemetry band. The translated signal is then retransmitted to a ground receiving station, where it is time-tagged and processed or recorded for later processing.

Oftentimes an application will require the use of a receiver-based user segment. For instance, using GPS for on-board navigation usually demands a receiver. On the other hand, when using a GPS user segment as a navigation reference for testing inertial navigation systems, a translator-based segment offers several advantages, including low cost, weight, and power consumption and high reliability. Furthermore, ground postprocessing of the signals allows for the use of highly accurate satellite orbital information not available in real-time and the use of highly detailed corrections. It also allows analysts to iteratively edit the data and respond to anomalous conditions. The result is accuracy better than that achievable by a receiver doing real-time navigation. 
The performance factors that affect the design of a GPS user segment are considered here. Models presented previously in the literature (and referenced throughout this work) are used to predict GPS performance as quantified by several specific measures. Although the results apply to various other applications, GPS used as a navigation reference for testing inertial navigation systems is considered as a specific example.

The inertial navigation system is a key component of aircraft, missiles, sounding rockets, launch vehicles, and other aerospace systems. It generally comprises three or more accelerometers, three or more gyros, and associated hardware and electronics. The inertial instruments (the accelerometers and gyros, known collectively as the inertial measurement unit or IMU) provide the navigation computer with the acceleration and attitude data necessary to generate velocity and position information [3]. The velocity and position data are in turn used by the guidance and control computer to achieve mission objectives, such as intercepting a target or inserting a payload into orbit. Errors in the IMU data result in errors in the navigated state and hinder the achievement of these objectives.

Flight testing is an important tool in evaluating the contribution of the IMU to errors in the navigated state. Estimating the source and magnitude of the IMU errors requires a separate trajectory reference. In the past, ground-based radars or a second on-board IMU have been used to provide the reference. The second option is usually prohibitive in terms of both cost and payload restrictions, while radars suffer from limitations in both geometry and accuracy. Recent flight-testing has demonstrated that a GPS user segment can provide a small, light, affordable, and accurate trajectory reference system for evaluating IMU errors [8].

Section II describes how the GPS user segment design affects the ability to meet user requirements. Important features of a user segment design, as well as their effect on GPS data quality, are described. The GPS error model used in the study is presented. Also included is an outline of the methods used to flight-test IMUs and the manner in which the data are processed.

Several different measures can be used to assess how well the user segment is performing. The measures may quantify one-dimensional or three-dimensional accuracy, or they may reflect the ability to meet overall user requirements. Section III defines these measures, including those used in flight-testing IMUs.

Parametric studies were performed to assess the sensitivity of the instrumentation system performance to the GPS user segment design. Section IV presents results which can be used to determine the complexity of a user segment required to achieve given GPS navigation accuracies as well as the broader flight-test objectives.

Finally, conclusions are presented in Section V. Included is a discussion of how the design curves

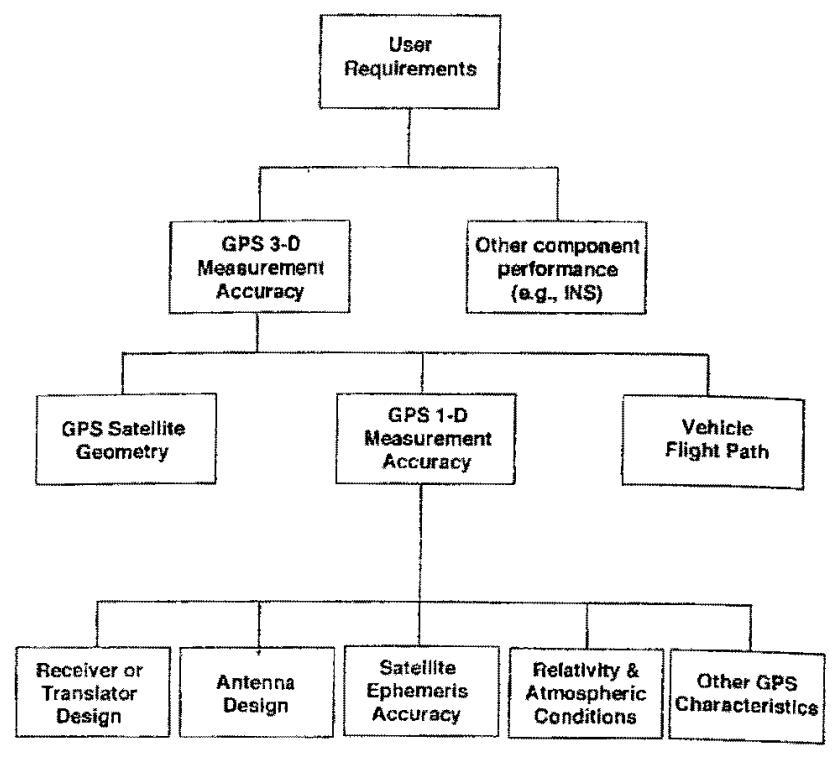

Fig. 1. User segment design.

developed in the previous sections can be used for other applications of GPS.

\section{USER REQUIREMENTS AND THE GPS USER SEGMENT}

\section{A. Contributors to System Performance}

The GPS user segment functions as part of a system designed to achieve some application-specific objective. The ability to meet performance requirements which quantify that objective depends on the design of the user segment as well as other factors relating to the system performance. This relationship is illustrated in Fig. 1. Each box represents either a measure of performance or a factor affecting performance; boxes higher in the figure depend on boxes connected to them from below.

At the top of the figure is the user's requirement on the performance of the whole system. For GPS/inertial navigation system hybrids, the user's requirement would typically be on the total navigation accuracy. For an autonomous GPS navigation system, the user requirement would be on the GPS navigation accuracy. For the case of GPS used as a trajectory reference for flight-testing inertial navigation systems, the user requirement would be on measures, such as estimation uncertainties, of the ability to estimate the errors of the system.

The three-dimensional measurement accuracy of the GPS user segment can be determined independently of other components in the user's system, as illustrated in the second level of Fig. 1. It depends on the satellite geometry, the vehicle flight path, and the one-dimensional GPS measurement accuracy [9]. In general for a GPS receiver, the measurements are in the form of satellite-to-receiver 
TABLE I

Ionospheric Refraction Correction

\begin{tabular}{|l|c|c|}
\hline \multirow{2}{*}{} & \multicolumn{2}{|c|}{$1-\sigma$ Accuracy (feet) (Gauss-Markov) } \\
\cline { 2 - 3 } & Range $\left(X_{\text {RA1 }}\right)$ & Delta Range $\left(X_{\text {Dal }}\right)$ \\
\hline Single Frequency & 25. & 1.6 \\
\hline Dual Frequency & 8. & 0.02 \\
\hline
\end{tabular}

Note: $\tau_{R A I}^{i}=10^{12} \mathrm{~s}_{i}^{*} \tau_{D R I}^{i}=200 \mathrm{~s}$.

Roman symbols here correspond to italic symbols in text.

TABLE II

Measurement Correction Parameters

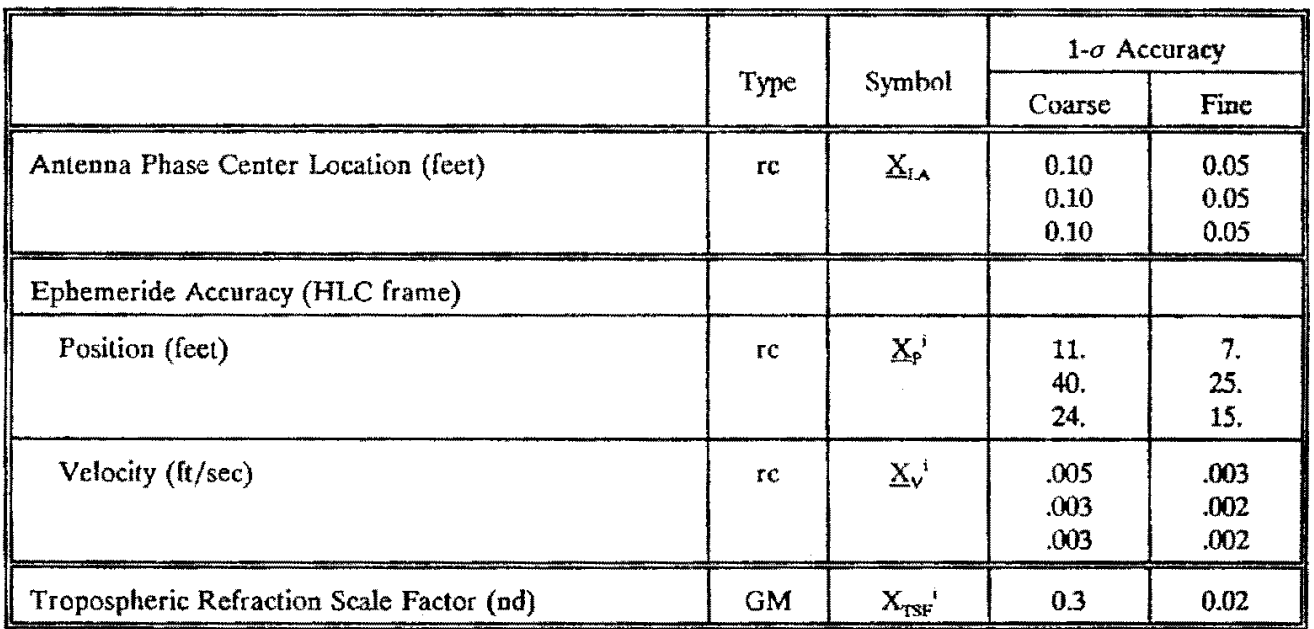

Note: $\mathrm{rc}=$ random constant, $\mathrm{GM}=$ Gauss-Markov process. $\tau_{\text {TSF }}^{i}=2000 \mathrm{~s}$.

Underlined symbols here correspond to boldface symbols in text.

Roman symbols here correspond to italic symbols in text.

range, determined from the codes modulated onto the GPS signal, and the change in that range (i.e., delta range) as derived from the phase of the GPS signal. For a GPS translator, the measurements are in the form of satellite-to-receiver-to-ground recorder range and delta range. The measurement accuracy depends on the receiver or translator design $[10,11]$, the antenna design, the accuracy of the satellite ephemeris data, relativity and atmospheric effects, and fixed characteristics of GPS [12]. Contributors to GPS accuracy are summarized in Tables I and II. The data are based on the literature $[13,14]$ and flight-test experience.

Receivers and translators can be designed to process the $L_{1}(1575.42 \mathrm{MHz})$ or $L_{2}(1227.60 \mathrm{MHz})$ signals or both. Processing two frequencies allows for better ionospheric refraction corrections, as shown in Table I [1]. In addition, receivers and translators can be designed to process one or both of the GPS codes. The GPS $L_{1}$ signal is quadrature modulated by two pseudorandom codes, a $1.023 \mathrm{Mbit} / \mathrm{s}$ coarse/acquisition (C/A) code and a $10.23 \mathrm{Mbit} / \mathrm{s}$ precision (P) code [7]. The type of code used determines the range precision which can be achieved. Note that the required bandwidth is $2 \mathrm{MHz}$ for the $\mathrm{C} / \mathrm{A}$ code and $20 \mathrm{MHz}$ for the P code.

The design and calibration of the antenna affects the accuracy of the phase-derived delta range measurement. The antenna phase induces error through three different mechanisms, as discussed in Section IIB. The accuracy of the phase center calibration also affects the calculation of vehicle reference point to phase center lever arm, effectively introducing measurement errors. Table II presents the phase center location uncertainty for both a fine and coarse calibration.

The GPS satellite ephemerides are obtained either in real-time from the GPS navigation message [15] or from satellite tracking data spanning a period of several days both before and after the time of interest. The accuracy of the ephemerides can be expressed as position and velocity standard deviations in height, long-track, cross-track (HLC) coordinates; Table II presents the values used for this study [13]. The HLC coordinate frame is a right-handed, noninertial coordinate system rotating with the satellite orbital motion. The first axis is parallel to a line segment connecting the Earth's center and the satellite; the 
TABLE III

User Segment Configurations

\begin{tabular}{|c|c|c|c|}
\hline Configuration & Codc & Frequency & Meas. Calc. \\
\hline A & $P$ & dual & fine \\
\hline B & $P$ & dual & coarse \\
\hline C & C/A & singlc & finc \\
\hline$D$ & C $/ A$ & single & coarse \\
\hline
\end{tabular}

third is parallel to the satellite orbital angular velocity vector. The second axis completes the orthogonal set.

Two different data correction schemes are considered. The coarser correction scheme adjusts the GPS measurements for satellite clock phase and frequency, drift in the translator carrier frequency, and changes in the signal path length due to ionospheric and tropospheric refraction. A coarse correction for relativistic effects is also built into the GPS clock frequency. A finer approach does the coarse corrections plus precise corrections for general and special relativistic effects due to the vehicle motion and higher accuracy tropospheric refraction corrections based on weather data. These corrections are summarized in Tables II and III. Fixed characteristics of GPS include the satellite clock phase and frequency accuracy after correction. Contributing to the GPS delta range resolution are the carrier wavelength, errors in the phase tracking loop, and atmospheric effects.

\section{B. GPS Error Model}

The various contributors to GPS measurement errors were modeled and then simulated to assess their impact on the user segment performance. A description of the model used in the simulation follows. Although this model applies to a translator-based user segment, it can be used for receivers by taking the receive time and location to be coincident with the translation time and location.

The GPS range measurement is modeled as

$$
\begin{aligned}
R^{i}\left(t_{k}\right)= & r^{i}\left(t_{k}\right)+\mathbf{S}^{i}\left(t_{k}^{*}\right)^{T} C_{R H}^{i}\left(t_{k}^{* *}\right) \mathbf{X}_{P}^{i} \\
& +\left(t_{k}^{* *}-t_{0}\right) \mathbf{S}^{i}\left(t_{k}^{*}\right)^{T} C_{R H}^{i}\left(t_{k}^{* *}\right) \mathbf{X}_{V}^{i} \\
& +\left(t_{k}^{* *}-t_{0}\right) X_{C F}^{i}+c / 10^{9} X_{C P}^{i}+B^{i}\left(t_{k}\right) X_{T S F}^{i}\left(t_{k}\right) \\
& +X_{R A I}^{i}\left(t_{k}\right)+\mathbf{S}^{i}\left(t_{k}^{*}\right)^{T} C_{R B}\left(t_{k}^{*}\right) \mathbf{X}_{L A}+v_{R}^{i}\left(t_{k}\right)
\end{aligned}
$$

where

$R^{i}$ is the measured range from the $i$ th satellite to the vehicle to the ground;

$r^{i}$ is the true range;

$t_{k}$ is the ground receive time;

$t_{k}^{*}$ is the vehicle translation time; $t_{k}^{* *}$ is the satellite transmission time;

$t_{0}$ is the reference time;

$\mathbf{S}^{i}$ is the unit vector from the vehicle to the $i$ th satellite;

$C_{R H}^{i}$ is the direction cosine matrix from the HLC frame for the $i$ th satellite to the reference frame;

$C_{R B}$ is the direction cosine matrix from the vehicle body frame to the referene frame;

$c$ is the speed of light;

$B^{i}$ is the tropospheric refraction correction for the $i$ th satellite;

$\mathbf{X}_{P}^{i}, \mathbf{X}_{V}^{i}, X_{C F}^{i}, X_{C P}^{i}, X_{T S F}^{i}$, and $X_{R A I}^{i}$ are per-satellite GPS errors (see Tables I-II);

$\mathbf{X}_{L A}$ are global GPS errors (see Table II);

$v_{R}^{i}$ is the range measurement noise for the $i$ th satellite.

Values for $B^{i}$ vary from $100 \mathrm{ft}$ on the ground to zero above approximately $50 \mathrm{mi}$ altitude. The $1 \sigma$ value for satellite clock phase error $X_{C P}^{i}$ is $10 \mathrm{~ns}$; the $1 \sigma$ value for satellite clock frequency error $X_{C F}^{i}$ is one part in $10^{12}$. The $1 \sigma$ values for $v_{R}^{i}$ are $25 \mathrm{ft}$ for $\mathrm{C} / \mathrm{A}$ code and $5 \mathrm{ft}$ for $P$ code.

The GPS delta range measurement is modeled as follows.

$$
\begin{aligned}
D^{i}\left(t_{k}\right)= & d^{i}\left(t_{k}\right)+\left[\mathbf{S}^{i}\left(t_{k}^{*}\right)^{T} C_{R H}^{i}\left(t_{k}^{* *}\right)\right. \\
& \left.-\mathbf{S}^{i}\left(t_{k-1}^{*}\right)^{T} C_{R H}^{i}\left(t_{k-1}^{* *}\right)\right] \mathbf{X}_{P}^{i} \\
& +\left[\left(t_{k}^{* *}-t_{0}\right) \mathbf{S}^{i}\left(t_{k}^{*}\right)^{T} C_{R H}^{i}\left(t_{k}^{* *}\right)\right. \\
& \left.\quad-\left(t_{k-1}^{* *}-t_{0}\right) \mathbf{S}^{i}\left(t_{k-1}^{*}\right)^{T} C_{R H}^{i}\left(t_{k-1}^{* *}\right)\right] \mathbf{X}_{V}^{i} \\
& +\left(t_{k}^{* *}-t_{k-1}^{* *}\right) X_{C F}^{i}+B^{i}\left(t_{k}\right) X_{T S F}^{i}\left(t_{k}\right) \\
& -B^{i}\left(t_{k-1}\right) X_{T S F}^{i}\left(t_{k-1}\right) \\
& +X_{D R I}^{i}\left(t_{k}\right)-X_{D R I}^{i}\left(t_{k-1}\right) \\
& +X_{D R A}^{i}\left(t_{k}\right)-X_{D R A}^{i}\left(t_{k-1}\right) \\
& +\left[\mathbf{S}^{i}\left(t_{k}^{*}\right)^{T} C_{R B}\left(t_{k}^{*}\right)-\mathbf{S}^{i}\left(t_{k-1}^{*}\right)^{T} C_{R B}\left(t_{k-1}^{*}\right)\right] \mathbf{X}_{L A} \\
& +X_{G R}^{i}\left(t_{k}\right)+X_{S R}^{i}\left(t_{k}\right)+v_{A C}^{i}\left(t_{k}\right)-v_{A C}^{i}\left(t_{k-1}\right)
\end{aligned}
$$

where

$D^{i}\left(t_{k}\right)$ is the measured delta range from the $i$ th satellite to the vehicle to the ground over the interval $\left(t_{k-1}, t_{k}\right]$;

$d^{i}\left(t_{k}\right)$ is the true delta range; 
$X_{D R I}^{i}$ and $X_{D R A}^{i}$ are per-satellite GPS errors (see Table I);

$X_{G R}^{i}$ and $X_{S R}^{i}$ are residual general and special relativity effects;

$v_{A C}^{i}\left(t_{k}\right)-v_{A C}^{i}\left(t_{k-1}\right)$ is the one-step anticorrelated delta range measurement noise for the $i$ th satellite;

$v_{A C}^{i}\left(t_{k}\right)$ is white and Gaussian and its $1 \sigma$ value is $0,031 \mathrm{ft}$.

The general and special relativity effects can be corrected quite accurately, and so the residual effects, $X_{G R}^{i}$ and $X_{S R}^{i}$, are assumed to be zero.

The GPS errors $X_{n}^{i}$ and $X_{n}$ (where $n=T S F, L A$, etc.) are assumed to be constants, random constants, or random variables from a first-order Gauss-Markov process. For the random constants,

$$
\begin{aligned}
X_{n}\left(t_{k+1}\right) & =X_{n}\left(t_{k}\right)=X_{n} \\
E\left[X_{n}\right] & =0 \\
E\left[X_{n}^{2}\right] & =\sigma_{n}^{2} .
\end{aligned}
$$

Values for $\sigma_{n}^{2}$ are given in Tables I-II. For the Gauss-Markov errors,

$$
\begin{aligned}
X_{n}\left(t_{k+1}\right) & =\exp \left(-\left(t_{k+1}-t_{k}\right) / \tau_{n}\right) X_{n}\left(t_{k}\right)+w_{n}\left(t_{k}\right) \\
E\left[X_{n}\left(t_{0}\right)\right] & =0 \\
E\left[X_{n}\left(t_{0}\right)^{2}\right] & =\sigma_{n}^{2}
\end{aligned}
$$

where $w_{n}$ is white Gaussian noise with

$$
E\left[w_{n}\left(t_{k}\right)^{2}\right]=\sigma_{n}^{2}\left[1-\exp \left(-2\left(t_{k+1}-t_{k}\right) / \tau_{n}\right)\right] .
$$

Values for $\sigma$ and $\tau$ are given in Tables I-II. For all random terms,

$$
E\left[X_{n}\left(t_{0}\right) X_{m}\left(t_{0}\right)\right]=0 .
$$

The antenna phase induces a Doppler range error through three mechanisms: error in the phase calibration, vehicle attitude error coupled with the antenna phase slope, and ionospheric refraction correction error.

The error due to antenna phase calibration is

$$
X_{D R A a}=\lambda e_{\mathrm{APC}} / 360
$$

where $\lambda$ is the wavelength of the signal, and $e_{\mathrm{APC}}$ is the antenna phase calibration error in degrees.

In general, the attitude of the aerospace vehicle is not perfectly known. Since the antenna phase pattern is typically a function of antenna orientation, the attitude error couples with the phase slope to produce an error given by

$$
X_{D R A b}=m \lambda e_{\mathrm{ATT}} / 360
$$

where $m$ is the antenna phase slope in degrees per degree, and $e_{\mathrm{ATT}}$ is the attitude error in degrees. This contribution is typically small; if the antenna phase slope is zero or if the vehicle attitude is perfectly known, it is zero.

The difference between the $L_{1}$ and $L_{2}$ phase measurements is used to determine and correct for the ionospheric refraction. Since the antenna phase calibration error is different for the two frequencies, the resulting ionospheric correction is in error. Starting from equations describing the ionospheric correction [1], the error can be shown to be

$$
X_{D R A c}=\frac{\lambda f_{2}\left(e_{\mathrm{APC} 1}-e_{\mathrm{APC} 2}\right)}{360\left(f_{1}-f_{2}\right)}
$$

where $f_{1}=(154)(10.23 \mathrm{MHz})=1672.42 \mathrm{MHz}$, the $L_{1}$ frequency; $f_{2}=(120)(10.23 \mathrm{MHz})=1227.60 \mathrm{MHz}$, the $L_{2}$ frequency; $e_{\mathrm{APC1}}, e_{\mathrm{APC}}$ are the antenna phase calibration errors for $L_{1}$ and $L_{2}$.

Assuming that the delta range is derived from the phase of $L_{1}$ and that attitude errors are negligible, the error is

$$
X_{D R A}=\frac{\lambda_{1} e_{\mathrm{APC} 1}}{360}+\frac{\lambda f_{2}\left(e_{\mathrm{APC} 1}-e_{\mathrm{APC} 2}\right)}{360\left(f_{1}-f_{2}\right)} .
$$

Taking the antenna phase calibration error to be Gaussian and

$$
\begin{aligned}
E\left[e_{\mathrm{APC} 1}^{2}\right] & =E\left[e_{\mathrm{APC} 2}^{2}\right]=\sigma_{\mathrm{APC}}^{2} \\
E\left[e_{\mathrm{APCl} 1} e_{\mathrm{APC} 2}\right] & =0.5 \sigma_{\mathrm{APC}}^{2}
\end{aligned}
$$

then, for dual frequency,

$$
E\left[X_{D R A}^{2}\right]=(0.00547)^{2} \sigma_{\mathrm{APC}}^{2}
$$

If single frequency GPS is used, then the antenna phase calibration does not contribute any ionospheric correction error to the delta range measurement. In this case,

$$
E\left[X_{D R A}^{2}\right]=(0.00174)^{2} \sigma_{\mathrm{APC}}^{2}
$$

Note for the single frequency case that even though the antenna phase portion of the ionospheric refraction correction error vanishes, the total ionospheric refraction error increases (see Table I).

\section{Flight-Testing Inertial Navigation Systems}

For the particular application of GPS used as a navigation reference for flight-testing IMUs, the user requirements are on the ability to estimate the total navigation error due to the IMU, the contribution of each of the major IMU error groups, and the contribution of individual IMU errors. The ability to estimate errors depends on the GPS user segment and the capability of the IMU (see Fig. 1).

To estimate IMU errors the IMU telemetry is processed with the GPS measurement data to generate observations that are functions of the IMU errors and the GPS errors. Specifically, the corrected GPS range and delta range are differenced with the equivalent quantities as indicated by the IMU under test. These 
IMU-indicated ranges and delta ranges are determined by using integrated accelerometer data and the satellite ephemerides. The GPS minus IMU-indicated ranges and delta ranges are used as the observations for a Kalman filter. The Kalman filter state vector contains an element for each modeled IMU and GPS error. The IMU errors are modeled as random constants:

$$
\begin{aligned}
X_{\mathrm{IMU}_{n}\left(t_{k+1}\right)} & =X_{\mathrm{IMU}_{n}}\left(t_{k}\right)=X_{\mathrm{IMU} n} \\
E\left[X_{\mathrm{IMU} n}\right] & =0 \\
E\left[X_{\mathrm{IMU} n}^{2}\right] & =\sigma_{\mathrm{IMU} n}^{2} .
\end{aligned}
$$

The observation matrix for the Kalman filter is derived from error models for accelerometers, gyros, initial conditions, and GPS. The IMU error models are propagated according to

$$
\begin{aligned}
& \frac{d}{d t} \frac{\partial r}{\partial X_{\mathrm{IMU} n}}=\frac{\partial v}{\partial X_{\mathrm{IMU} n}} \\
& \frac{d}{d t} \frac{\partial v}{\partial X_{\mathrm{IMU} n}}=\frac{\partial g}{\partial r} \frac{\partial r}{\partial X_{\mathrm{IMU} n}}+a \times \frac{\partial \Psi}{\partial X_{\mathrm{IMU} n}}+f_{\mathrm{IMU} n} \\
& \frac{d}{d t} \frac{\partial \Psi}{\partial X_{\mathrm{IMU} n}}=f_{\mathrm{IMU} n}
\end{aligned}
$$

where $r, v$, and $\Psi$ are the inertial navigation system errors in position, velocity, and attitude and $f_{\mathrm{IMU} n}$ are forcing functions for each accelerometer, gyro, and initial condition error. These equations are integrated numerically and transformed into GPS observation coordinates to produce the sensitivities of the filter observations to IMU errors $[3,16]$. The sensitivities of the filter observations to GPS errors are determined from the GPS error model presented above.

\section{GPS USER SEGMENT PERFORMANCE MEASURES}

\section{A. General Performance Measures}

The performance of a GPS user segment, including its ability to achieve user objectives, can be quantified using various measures. Measures of the ability to meet user requirements are application specific. On the other hand, measures of performance at lower levels in the system design can be defined without reference to the specific application.

The GPS three-dimensional measurement accuracy can be quantified by a six by six position/velocity error covariance matrix. Although the position and velocity measurement errors are correlated (because the range and delta range errors are correlated), the position-to-velocity covariances are small compared with the position and velocity variances, the position-to-position covariances, and the velocity-to-velocity covariances. Therefore, the position-to-velocity covariances are ignored and the accuracy is expressed instead as two smaller matrices, each three by three. These GPS position and velocity error covariance matrices $P_{\text {POS }}$ and $P_{\mathrm{VEL}}$ are calculated by propagating the GPS error variances into position/velocity space.

Because $P_{\text {POS }}$ and $P_{V E L}$ are matrices and therefore rather unwieldly, a scalar representation of measurement accuracy derived from them, known as the spherical error probable (SEP), is used instead. The SEP is defined as the 50th percentile probability radius,

$$
\begin{gathered}
\int_{0}^{\mathrm{SEP}} \int_{0}^{\pi} \int_{0}^{2 \pi} f_{3}(x, y, z) r^{2} \sin \phi d \Theta d \phi d r=0.5 \\
x=r \sin \phi \cos \Theta \\
y=r \sin \phi \sin \Theta \\
z=r \cos \phi
\end{gathered}
$$

where $f_{3}$ is a trivariate Gaussian probability density function. The trivariate density function $(n=3)$ is a special case of the general multivariate density function

$$
\begin{aligned}
f_{n}\left(x_{1}, x_{2}, \ldots, x_{n}\right)= & (2 \pi)^{-n / 2}|P|^{-1 / 2} \\
& \times \exp \left[-1 / 2(\mathbf{x}-\mathbf{m})^{T} P^{-1}(\mathbf{x}-\mathbf{m})\right] \\
E[\mathbf{x}]= & \mathbf{m} .
\end{aligned}
$$

It is assumed that

$$
\mathbf{m}=0 .
$$

One SEP each can be calculated from the position and velocity covariance matrices [17]; smaller SEPs indicate better GPS performance.

The GPS one-dimensional measurement accuracy can be expressed as two (scalar) standard deviations, one each for range and delta range. These one-dimensional accuracies are calculated by propagating GPS error variances into range and delta range space. Smaller numbers represent better performance.

The GPS satellite geometry is usually quantified by the Geometric Dilution of Precision. Because the GPS constellation provides uniformly good geometry, this study did not vary the assumed satellite geometry; a full constellation was used in the simulations.

\section{B. Performance Measures for IMU Flight-Testing}

Several different measures can be used to quantify the ability to estimate IMU errors given the GPS data. One important measure is the total estimation uncertainty. This information is produced by the filter in the form of a large covariance matrix, a square matrix with a side dimension equal to that of the filter state. Because the uncertainty in this form is very unwieldy, a preferable measure is a circular error 


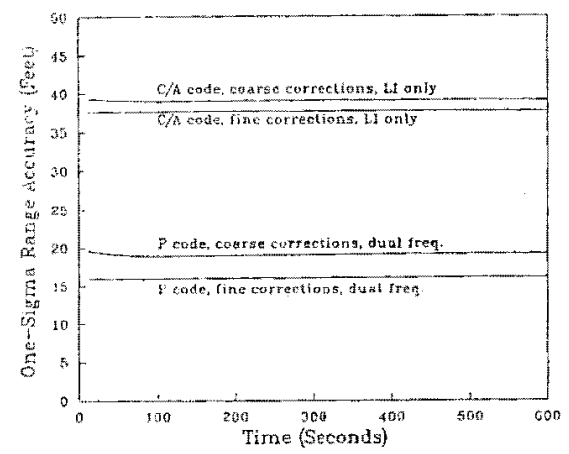

Fig. 2. GPS range measurement accuracy.

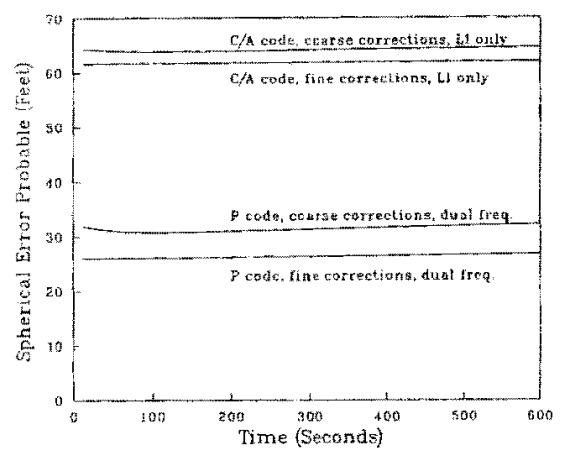

Fig. 3. GPS position SEP.

probable (CEP) based on it. This scalar is produced by first propagating the state space error covariance matrix into deployment position/velocity space and then into impact space. The required transitions are generated numerically from a model of the error propagation. Once in impact space, the error covariance is used to calculate a 50 th percentile radius $[18,19]$ :

$$
\begin{aligned}
\int_{0}^{\mathrm{CEP}} \int_{0}^{2 \pi} f_{2}(x, y) r d \Theta d r & =0.5 \\
x & =r \cos \Theta \\
y & =r \sin \Theta
\end{aligned}
$$

where $f_{2}$ is the bivariate Gaussian density function $(n=2)$. It is assumed that

$$
\mathbf{m}=0 \text {. }
$$

Other measures of the filter performance given the GPS data are the group estimation uncertainties, also indicated by CEPs. These quantities are calculated in a fashion similar to the total estimation uncertainty CEP, except they are based only on certain submatrices of the error covariance matrix corresponding to the accelerometer, gyro, or initial condition (including IMU clock) error groups.

The performance of the filter can also be measured by the individual error state recovery ratios. The recovery ratio for an error state is the final standard deviation of a state estimate divided by the initial standard deviation. It represents the ratio of final

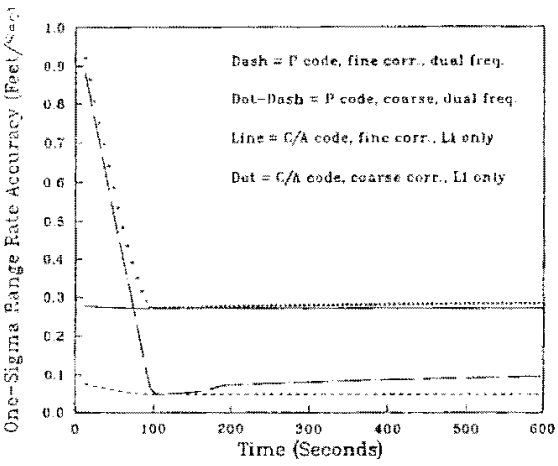

Fig. 4. GPS range rate measurement accuracy (antenna error $=50^{\circ}$ )

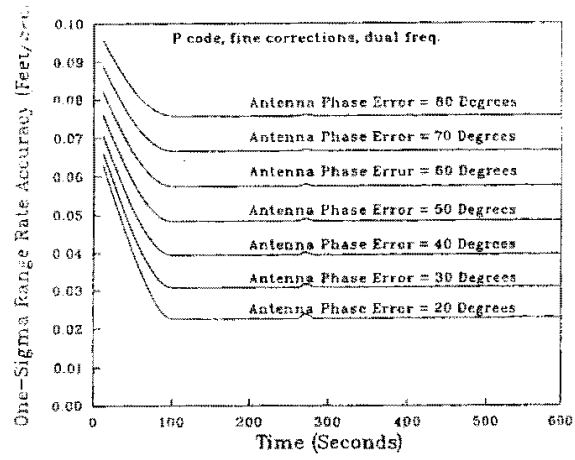

Fig. 5. GPS range rate measurement accuracy.

uncertainty to initial uncertainty for an individual error state; low recovery ratios indicate better estimates. Rather than tabulate the recovery ratios for every error state, the measure used is the fraction of states having a recovery ratio exceeding 0.5 .

\section{GPS USER SEGMENT DESICN ANALYSIS}

An analysis of a GPS user segment was performed to determine the effects on performance of the design parameters described in Section II; the results of the analysis are presented in this section. These results can be used to determine the basic design parameters for a GPS user segment needed to achieve a desired performance.

Four fundamental design parameters were varied in the analysis: the code type (C/A or P), the number of frequencies (single or dual), the measurement correction scheme (coarse or fine), and the antenna phase calibration error standard deviation $\sigma_{\mathrm{APC}}$ (from 20 to $80 \mathrm{deg}$ in ten deg increments). Four user segment configurations, representing various combinations of code type, frequency usage, and measurement correction scheme, were studied, as shown in Table III. Furthermore, the antenna phase calibration error was varied for one of the configurations. Figs. 2-9 show the results of the analysis.

The one-dimensional results (Figs. 2, 4, and 5) do not depend on either the vehicle motion or the specific 


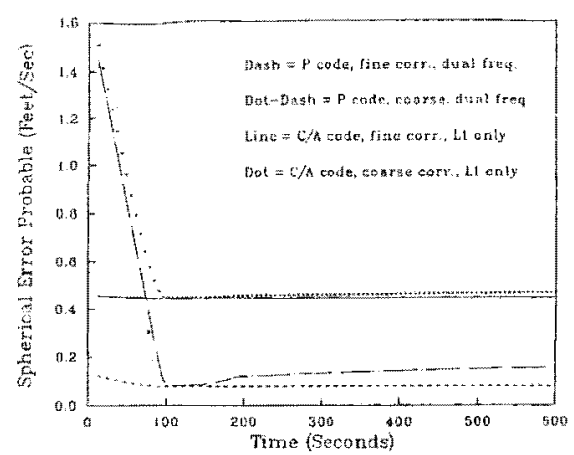

Fig. 6. GPS velocity SEP (antenna error $=50^{\circ}$ ).

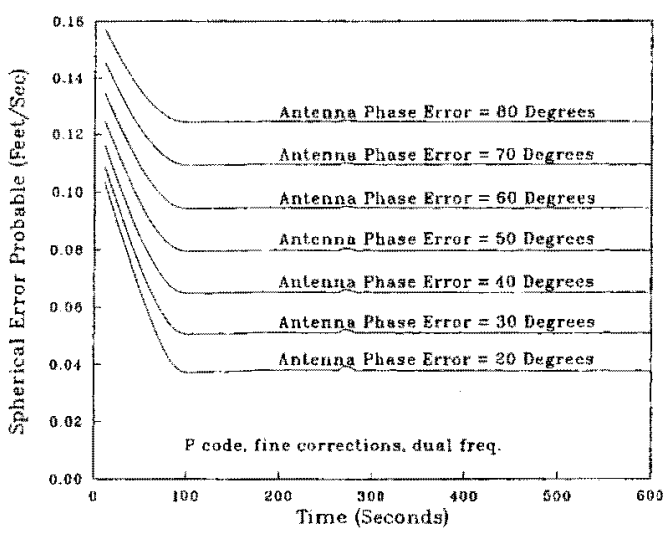

Fig. 7. GPS velocity SEP.

user application, other than the assumption that conditions permit the user segment to produce useful measurements. The three-dimensional results (Figs. 3, 6 , and 7) depend on the relative motion between the vehicle and the GPS satellites. The analysis considered a three-stage missile on a $4000 \mathrm{~m}$ trajectory. Peak acceleration during the $54 \mathrm{~s}$ first stage is $5.7 \mathrm{~g}$; during the $72 \mathrm{~s}$ second stage, $3.5 \mathrm{~g}$; and during the $54 \mathrm{~s}$ third stage, $7.1 \mathrm{~g}$. Velocity at the end of the $180 \mathrm{~s}$ boost is $12600 \mathrm{ft} / \mathrm{s}$, while the altitude is 165 miles. Nominal satellite coverage is assumed.

Figs. 8 and 9 are for the specific case of GPS as a trajectory reference for evaluating inertial navigation system errors. The inertial navigation systems considered had IMUs with the characteristics listed in Table IV. The IMU model for this study contained a total of 76 terms, including 33 accelerometer terms, 33 gyro terms, nine initial condition terms, and one

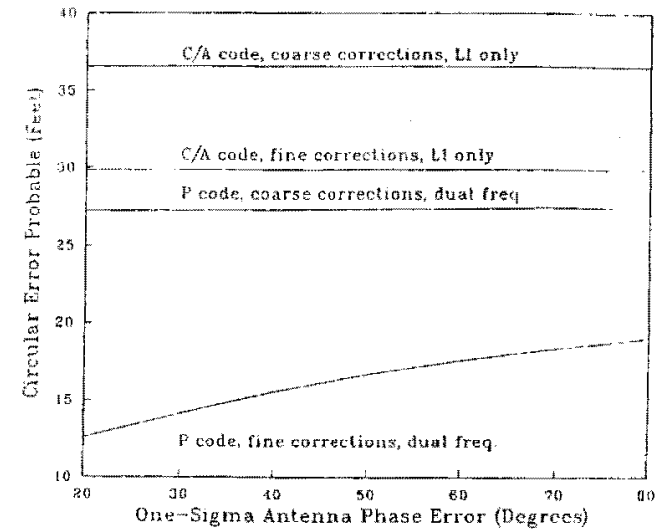

Fig. 8. Total estimation error for IMU I.

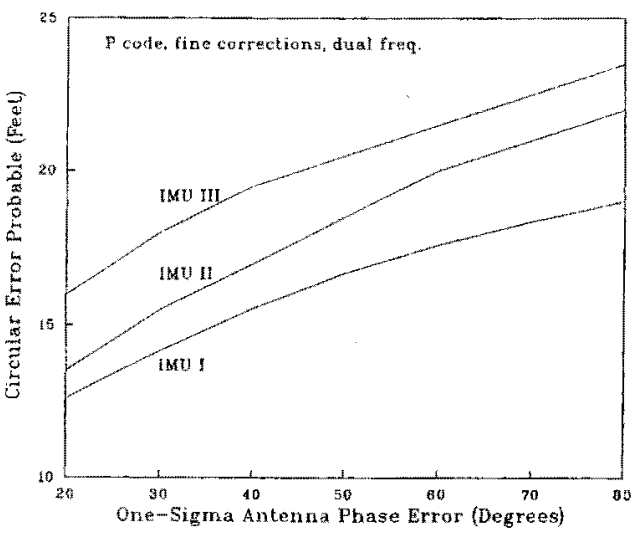

Fig. 9. Total estimation error for various IMUs.

IMU clock frequency error (grouped with the initial condition terms for convenience). The IMU errors are modeled as being initially random (Gaussian with zero mean and some assumed standard deviation) but constant throughout the flight. The effects of the errors on the navigated state and the GPS observations are functions of time [8]. The standard deviations of the random IMU errors were transformed into impact space and expressed as CEPs in Table IV. Configuration A (Table III) was considered for all three IMUs, while configurations B, C, and D were considered for IMU I only.

Figs. 2 and 3 contain the one- and three-dimensional position accuracies for the four configurations. Figs. 4-7 present the one-dimensional

TABLE IV

IMU Capabilities

\begin{tabular}{|l|c|c|c|}
\hline \multirow{2}{*}{} & \multicolumn{3}{|c|}{ CEP (feet) } \\
\cline { 2 - 4 } & Class I & Class II & Class III \\
\hline Accelerometer errors & 304 & 3040 & 30400 \\
\hline Gyro errors & 164 & 1640 & 16400 \\
\hline Initial Condition Errors & 231 & 2310 & 23100 \\
\hline \hline Total IMU & 500 & 5000 & 50000 \\
\hline
\end{tabular}


range rate accuracies and the three-dimensional velocity accuracies. The largest effects on GPS measurement accuracy are the code type, which establishes the range resolution, and whether a second frequency is used for ionospheric corrections. Range rate measurements with coarse corrections also contain large residual refraction errors during the first $100 \mathrm{~s}$ of flight, within the troposphere. As seen in Figs. 5 and 7, the antenna phase calibration error also has a significant effect on range rate for the dual frequency configurations; otherwise, its contribution gets swamped by the ionosphere refraction error. Note that the antenna phase error does not affect the range measurement (see Section IIB).

Fig. 8 shows the effects of the user segment configuration on the ability to estimate the total IMU navigation error, for a class I IMU. Refraction errors (ionospheric for the $L_{1}$ only configuration, tropospheric for the coarse correction case) are seen to degrade the ability to estimate the total error due to the IMU. On the other hand, the antenna phase error is important only if the measurements are derived from dual frequency $P$ code using fine corrections.

The ability to estimate the errors of different IMUs, for configuration A (P code, dual frequency, fine corrections), is illustrated in Fig. 9. Note that the estimation uncertainty for the total IMU error is quite good for all the three classes of IMUs, but is somewhat dependent on antenna phase calibration error. On the other hand, the ability to estimate major IMU error groups is relatively limited, especially for less accurate IMUs. Even for Class I IMUs, the estimation uncertainty for the groups is several times higher than for the total. This indicates that GPS is able to estimate the total IMU performance quite well, but the estimation errors for the IMU error groups are highly correlated to one another. Finally, GPS is limited in its ability to discern individual IMU errors. Only a third to a half of the individual errors have estimation uncertainties significantly less (i.e., 50\%) than the a priori uncertainty. More individual errors can be recovered from less accurate IMUs where the effects of individual errors are larger.

The results shown in Figs. 2-9 can be used to design a GPS user segment based on user requirements. Consider the example of a GPS receiver to be used as an autonomous navigator with a SEP accuracy requirements of $30 \mathrm{ft}$ in position and $0.12 \mathrm{ft} / \mathrm{s}$ in velocity. A suitable design would use $P$ code, dual frequencies, and fine corrections (Fig. 3) and an antenna phase calibration with a $1 \sigma$ error of $45 \mathrm{deg}$ or less (Fig. 7). On the other hand, if the velocity SEP is important to the user only when his vehicle is outside the troposphere, he can use an antenna with a $70 \mathrm{deg}$ phase calibration error (Fig. 7).

Another example is a user who wants to estimate the errors in a class I IMU. Figs. 8 and 9 show what kind of error recovery is possible for different user segment configurations. If the requirement on estimation uncertainty CEP for the total IMU error is $40 \mathrm{ft}$, a single frequency $\mathrm{C} / \mathrm{A}$ code receiver using coarse corrections will suffice (Fig. 8). On the other hand, a requirement of $10 \mathrm{ft}$ cannot be met.

\section{SUMMARY}

The performance required of a GPS user segment depends on the application-dependent objectives. The performance is achieved by appropriate design of the measurement calculation scheme, the antenna, and the receiver or translator. Performance measures can be defined at various levels; each level takes into account various components of the overall application. The top level measures presented here are peculiar to the specific application of GPS as a navigation reference for testing IMUs, but the other measures are not. Therefore, the one- and three-dimensional accuracy data presented here can be used to design GPS user segments for a wide variety of applications.

The results of this study indicate that the ability to track $\mathrm{P}$ code on two different frequencies is the most critical aspect of GPS user segment design. In addition, the extra effort required to perform fine data corrections, especially tropospheric refraction corrections, results in a significant improvement in GPS accuracy. Antenna phase calibration is critical only if dual frequency tracking is used.

\section{REFERENCES}

[1] Spilker, J. J., Jr. (1980)

GPS signal structure and performance characteristics. Global Positioning System Papers, Vol. I.

The Institute of Navigation, Washington, DC, 1980, 29-54.

(2) Dougherty, J. J., El-Sherief, H., and Simon, D. J. (1993) Application of GPS for missile post flight guidance accuracy analysis.

In Proceedings of the First IEEE Regional Conference on Aerospace Control Systems, Institute of Electrical and Electronics Engineers, Piscataway, NJ, 1993, 777-781.

[3] Siouris, G. (1993)

Aerospace Avionics Systems: A Modern Synthesis. New York: Academic Press, 1993, ch. 1, 2, 3, 5, 6 .

[4] Simon, D., and El-Sherief, H. (1993) GPS receiver design for integrated navigation systems. In Proceedings of the Institute of Navigation $49 \mathrm{th}$ Annual Meeting, 1993, 437-445.

[5] Simon, D., and El-Sherief, H. (1993) Design of global positioning system receivers for integrated inertial navigation systems. In Proceedings of the IEEE Conference on Decision and Control, Institute of Electrical and Electronics Engineers, Piscataway, NJ, 1993, 1476-1477.

[6] Upadhyay, T., Cotterill, $S_{*}$, and Deaton, A. W. (1993) Autonomous GPS/INS navigation experiment for space transfer vehicle. IEEE Transactions on Aerospace and Electronic Systems, 29 (July 1993), 772-785. 
[7] Kao, M. H., and Eller, D. H. (1983)

Multiconfiguration Kalman filter design for high-performance GPS navigation.

IEEE Transactions on Automatic Control, AC-28 (1983), 304-314.

[8] Dougherty, J. J., El-Sherief, H, and Hohman, D. S. (1994) The use of GPS for evaluating inertial measurement unit errors.

Journal of Guidance, Control, and Dynamics, 17, 3 (1994), 435-441.

[9] Simon, D., and El-Sherief, $\mathrm{H}$.

Navigation satellite selection using neural networks. Neurocomputing -An Intemational Journal, to be published.

[10] Simon, D., and El-Sherief, H. (1994)

Hybrid $\mathrm{H} 2 / \mathrm{H}$-infinity estimation for phase locked loop filter design.

In Proceedings of the American Control Conference,

Institute of Electrical and Electronics Engineers,

Piscataway, NJ, 1994, 2212-2216.

[11] Simon, D., and El-Sherief, H. (1994)

Fuzzy logic for phase locked loops.

In Proceedings of the Position, Location and Navigation

Symposium, Institute of Electrical and Electronics

Engineers, Piscataway, NJ, 1994, 252-259.

[12] Conley, R. (1993)

GPS performance: What is normal?

Navigation: Joumal of the Institute of Novigation, 40 (Fall 1993), 261-281.

[13] U.S. Air Force (1991)

NAVSTAR GPS user equipment (public release version). U.S. Air Force Space Systems Division, NAVSTAR-GPS Joint Program Office, Los Angeles, CA, Feb. 1991.
[14] Janiczek, P, and Gilbert, S. (Eds.) (1980)

Global Positioning System Papers, Vol. I-III,

Washington, DC: The Institute of Navigation, 1980.

[15] Van Dierendonck, A. J., et al. (1980)

The GPS navigation message.

Global Positioning System Papers, Vol. I.

Washington, DC: The Institute of Navigation, 1980, 55-73.

[16] McAllister, D. F, and Wilcox, J. C. (1969)

Digital computer program for generalized inertial guidance system error analysis, Version 11, Modification 1.

TRW Systems Group, San Bernadino, CA, Mar. 1969

[17] Del Marco, S. (1993)

A series representation of the spherical error probability integral.

IEEE Transactions on Aerospace and Electronic Systems, 29 (Oct. 1993), 1349-1356.

[18] Gillis, J. T. (1991)

Computation of the circular error probability integral.

IEEE Transactions on Aerospace and Electronic Systems, 27 (Nov. 1991), 906-910.

[19] Rogers, S. R. (1993)

Comments on "Computation of the Circular Error Probability Integral".

IEEE Transactions on Aerospace and Electronic Systems, 29 (Apr. 1993), 553-555. 